\title{
THE MINIMAL SUPPORT FOR A CONTINUOUS FUNCTIONAL ON A FUNCTION SPACE
}

\author{
KAZUHIKO MORISHITA \\ (Communicated by Dennis Burke) \\ Dedicated to Professor Ryosuke Nakagawa on his sixtieth birthday
}

\begin{abstract}
Let $C_{p}(X)$ be the function space with the pointwise convergent topology over a Tychonoff space $X$ and $\xi$ a continuous real-valued function on $C_{p}(X)$. A closed subset $S$ of $X$ is called a support for $\xi$ if $\xi(f)=\xi(g)$ holds for any pair $(f, g)$ of elements of $C_{p}(X)$ such that $f_{\mid S}=g_{\mid S}$. It is proven that the minimal support for any real-valued continuous function on the space $C_{p}(X)$ exists.
\end{abstract}

\section{INTRODUCTION}

In this paper we assume that all spaces are Tychonoff. Let $C_{p}(X)$ be the space of all real-valued continuous functions on $X$ with the topology of pointwise convergence. We call a real-valued function on $C_{p}(X)$ a functional. For a family $\mathscr{A}$ of sets, we write $\bigcap \mathscr{A}=\bigcap\{A: A \in \mathscr{A}\}$. For a function $f$ on $X$ and a subset $M$ of $X$, the restriction of $f$ to $M$ is denoted by $f_{\mid M} . \mathbb{R}$ and $\mathbb{N}$ denote the real line and the set of natural numbers respectively. Other undefined terms can be found in [E].

Let $\xi$ be a continuous functional on $C_{p}(X)$. A subset $S$ of $X$ is called a quasi-support for $\xi$ if $\xi(f)=\xi(g)$ holds for any pair $(f, g)$ of elements of $C_{p}(X)$ such that $f_{\mid S}=g_{\mid S}$. And if a quasi-support $S$ is closed, then $S$ is called a support. We say that a support $S$ is minimal if for every support $T$ for $\xi$ that is contained in $S$ we have $S=T$. It is known [A] that a linear continuous functional $\xi$ on $C_{p}(X)$ is expressed as a linear combination of a finite subset of $X$. That is $\xi=\sum_{i=1}^{n} \alpha_{i} x_{i}$ for some finite subset $\left\{x_{1}, \ldots, x_{n}\right\}$ of $X$ and numbers $\left\{\alpha_{1}, \ldots, \alpha_{n}\right\}$. In this case the set $\left\{x_{1}, \ldots, x_{n}\right\}$ is clearly the minimal support for $\xi$. Our purpose of this paper is to give a general result for any continuous functional on $C_{p}(X)$ :

Theorem 0. There exists the minimal support $S$ for any continuous functional on $C_{p}(X)$ and $S$ is a separable subspace of $X$.

Received by the editors May 23, 1990 and, in revised form, September 19, 1990.

1980 Mathematics Subject Classification (1985 Revision). Primary 54C05, 54C30, 54C35.

Key words and phrases. Function space, functional, pointwise convergent topology, support. 


\section{Proof of Theorem 0}

We write

$$
\langle f, \Gamma, \varepsilon\rangle=\left\{g \in C_{p}(X):|f(x)-g(x)|<\varepsilon \text { for any } x \in \Gamma\right\}
$$

for a continuous function $f$ on $X$, a finite subset $\Gamma$ of $X$, and a positive number $\varepsilon . \pi_{M}: C_{p}(X) \rightarrow C_{p}(M)$ denotes the restriction map from $X$ to a subspace $M$. It is known [A] that if $M$ is a closed subset of $X$ then $\pi_{M}$ is an open map onto $\pi_{M}\left(C_{p}(X)\right)$. Let Supp $\xi$ be the set of all supports for a continuous functional $\xi$ on $C_{p}(X)$. To prove the theorem, we show some results. We may assume that $\xi$ is not constant.

Lemma 1. For any pair $(S, T)$ of elements of $\operatorname{Supp} \xi, S \cap T$ belongs to $\operatorname{Supp} \xi$.

Proof of Lemma 1. Put $A=S \cap T$. Assume that there exist continuous functions $f$ and $g$ on $X$ such that $\xi(f) \neq \xi(g)$ and $f_{\mid A}=g_{\mid A}$. It is easily checked the following equation

$$
\pi_{S}^{-1}\left(\pi_{S}\left(\xi^{-1}(\xi(g))\right)\right)=\xi^{-1}(\xi(g))
$$

for the restriction map $\pi_{S}$. From this, we have that $f_{\mid S}$ does not belong to $\pi_{S}\left(\xi^{-1}(\xi(g))\right)$. Since $S$ is closed, $\pi_{S}$ is open. We have that $\pi_{S}\left(\xi^{-1}(\xi(g))\right)$ is closed in $\pi_{S}\left(C_{p}(X)\right)$. There exist a finite subset $\Gamma$ of $S$ and a positive number $\varepsilon$ such that

$$
\langle f, \Gamma, \varepsilon\rangle \cap \xi^{-1}(\xi(g))=\varnothing .
$$

We consider the following three cases.

Case I. If $\Gamma \cap T=\varnothing$ holds, then there exists a continuous function $h$ on $X$ such that $h_{\mid \Gamma}=f_{\mid \Gamma}$ and $h_{\mid T}=g_{\mid T}$. We have that $h \in\langle f, \Gamma, \varepsilon\rangle$ and $\xi(h)=\xi(g)$ because $T$ is a support for $\xi$. This is a contradiction.

In the following two cases we assume that $\Gamma \cap T \neq \varnothing$ and put $\Gamma_{1}=\Gamma \cap T$ and $\Gamma_{2}=\Gamma \backslash \Gamma_{1}$.

Case II. If $\left\langle f, \Gamma_{1}, \varepsilon\right\rangle \cap \xi^{-1}(\xi(g)) \neq \varnothing$ holds, then we can take a function $h \in\left\langle f, \Gamma_{1}, \varepsilon\right\rangle \cap \xi^{-1}(\xi(g))$. There exists a continuous function $\tilde{h}$ on $X$ such that $\tilde{h}_{\mid T}=h_{\mid T}$ and $\tilde{h}_{\mid \Gamma_{2}}=f_{\mid \Gamma_{2}}$. Here we have that $\tilde{h} \in\langle f, \Gamma, \varepsilon\rangle$ and $\xi(\tilde{h})=$ $\xi(h)=\xi(g)$. This is a contradiction.

Case III. If $\left\langle f, \Gamma_{1}, \varepsilon\right\rangle \cap \xi^{-1}(\xi(g))=\varnothing$ holds, then we have $\pi_{A}\left(\left\langle f, \Gamma_{1}, \varepsilon\right\rangle\right) \cap$ $\pi_{A}\left(\xi^{-1}(\xi(g))\right)=\varnothing$ for the restriction map $\pi_{A}$ because $\Gamma_{1}$ is contained in $A$. However, by our assumption, we have

$$
\pi_{A}(f)=\pi_{A}(g) \in \pi_{A}\left(\left\langle f, \Gamma_{1}, \varepsilon\right\rangle\right) \cap \pi_{A}\left(\xi^{-1}(\xi(g))\right) .
$$

This is a contradiction. Lemma 1 is proved.

Lemma 2. $\cap \operatorname{Supp} \xi$ is a support for $\xi$.

Proof of Lemma 2. Put $S=\bigcap \operatorname{Supp} \xi$. Assume that there exist continuous functions $f$ and $g$ on $X$ such that $\xi(f) \neq \xi(g)$ and $f_{\mid S}=g_{\mid S}$. Since $f$ does not belong to a closed set $\xi^{-1}(\xi(g))$ in $C_{p}(X)$, there exist a finite subset $\Gamma$ of $X$ and a positive number $\varepsilon$ such that $\langle f, \Gamma, \varepsilon\rangle \cap \xi^{-1}(\xi(g))=\varnothing$. Using Lemma 1 , by the definition of $S$, we can find an element $T$ of Supp $\xi$ such that $T \cap(\Gamma \backslash S)=\varnothing$. Consider three cases in the same way as we did in the proof of Lemma 1. We have a contradiction in any case. The proof of the lemma is completed. 
Lemma 3. $\cap \operatorname{Supp} \xi$ is a separable subspace of $X$.

Proof of Lemma 3. Put $S=\bigcap \operatorname{Supp} \xi$. Since $S$ is a support for $\xi$ (Lemma $2)$, there exists a functional $\tilde{\xi}$ on $\pi_{S}\left(C_{p}(X)\right)$ such that $\xi=\tilde{\xi} \circ \pi_{S}$. Since $S$ is closed, $\pi_{S}$ is open. We have that $\tilde{\xi}$ is continuous. And the cellurality of the space $\pi_{S}\left(C_{p}(X)\right)$ is countable (See [A]). There exists a countable quasi-support $A$ for $\tilde{\xi}$ in $S$. Obviously $\mathrm{cl}_{X} A$ is a support for $\xi$. So $S=\operatorname{cl}_{X} A$ holds by the minimality of the support $S$ (Lemma 2). The lemma is proved.

\section{REMARKS AND COMMENTS}

I. For any countable subset $A$ of $X$ we can find a continuous functional $\xi$ on $C_{p}(X)$ such that $\bigcap \operatorname{Supp} \xi=\operatorname{cl} A$. To do this, we assume that $A$ is indexed as $A=\left\{x_{n}: n \in \mathbb{N}\right\}$. For every $f \in C_{p}(X)$, we put

$$
\xi(f)=\sum\left\{2^{-n} r\left(f\left(x_{n}\right)\right): n \in \mathbb{N}\right\},
$$

where $r: \mathbb{R} \rightarrow[0,1]$ is a continuous function defined by

$$
r(\alpha)= \begin{cases}1 & (1 \leq \alpha), \\ \alpha & (0 \leq \alpha \leq 1), \\ 0 & (\alpha \leq 0) .\end{cases}
$$

II. Using the same idea in the proof of our theorem, we can prove the following lemma.

Lemma 4. Let $\mathscr{F}$ be a nonempty proper closed subset of $C_{p}(X)$. We put $\operatorname{Supp} \mathscr{F}=\left\{S \subset X: S\right.$ is closed in $\left.X, \pi_{S}^{-1}\left(\pi_{S}(\mathscr{F})\right)=\mathscr{F}\right\}$. Then the set $\cap \operatorname{Supp} \mathscr{F}$ belongs to $\operatorname{Supp} \mathscr{F}$.

This lemma gives a result on the minimal support.

Theorem 5. Let $\xi$ be a nonconstant continuous functional on $C_{p}(X)$. For an $\alpha \in \xi\left(C_{p}(X)\right)$, we put $S_{\alpha}=\cap \operatorname{Supp} \xi^{-1}(\alpha)$. Then we have $\bigcap$ Supp $\xi=$ $\operatorname{cl}\left(\bigcup\left\{S_{\alpha}: \alpha \in \xi\left(C_{p}(X)\right)\right\}\right)$.

Proof. We can easily show the following two claims.

(1) For any $\alpha \in \xi\left(C_{p}(X)\right), \cap \operatorname{Supp} \xi \in \operatorname{Supp} \xi^{-1}(\alpha)$ holds.

(2) $\bigcup\left\{S_{\alpha}: \alpha \in \xi\left(C_{p}(X)\right)\right\}$ is a quasi-support for $\xi$.

We have $S_{\alpha} \subset \bigcap \operatorname{Supp} \xi$ for any $\alpha \in \xi\left(C_{p}(X)\right)$ by (1), and $\bigcap \operatorname{Supp} \xi \subset$ $\mathrm{cl}\left(\bigcup\left\{S_{\alpha}: \alpha \in \xi\left(C_{p}(X)\right)\right\}\right)$ by (2). The theorem is proved.

Added in proof. The author recently proved that the similar results hold for the compact-open topology but fail in general for the norm topology.

\section{REFERENCES}

[A] A. V. Arhangel'skii, Function spaces with the pointwise topology. I, General Topology, Function Spaces and Dimension, Moscow Univ., 1985, pp. 3-66. (Russian)

[E] R. Engelking, General topology, PWN, Warszawa, 1977.

Institute of Mathematics, University of Tsukuba, Tsukuba-shi, Ibaraki, 305, Japan 